\title{
Search for long distance time correlations between cosmic air showers with the MRPC telescopes of the EEE network
}
P. La Rocca ${ }^{\star a b}$,
M. Abbrescia ${ }^{a c}$
C. Avanzini ${ }^{a d}$, L. Baldini ${ }^{a d}$, R.
R. Baldini Ferroli ${ }^{a e}$,
G. Batignani ${ }^{a d}$, M. Battaglieri ${ }^{a f}$, S. Boi ${ }^{a g}$, E. Bossini ${ }^{a h}$, F. Carnesecchi ${ }^{a i}$, C. Cicalò ${ }^{a g}$, L. Cifarelli ${ }^{a i}$, F. Coccetti ${ }^{a}$, E. Coccia ${ }^{a j}$, A. Corvaglia ${ }^{a k}$, D. De Gruttola ${ }^{a l}$,
S. De Pasquale ${ }^{a l}$, F. Fabbri ${ }^{a e}$, L. Galante ${ }^{a m}$, P. Galeotti ${ }^{a m}$, M. Garbini ${ }^{a i}$, G. Gemme $^{a f}$, I. Gnesi ${ }^{a m}$, S. Grazzi ${ }^{a}$, D. Hatzifotiadou ${ }^{a i o}$, Z. Liu ${ }^{a o p}$, G. Mandaglio ${ }^{a q}$, G. Maron ${ }^{r}$, M. N. Mazziotta ${ }^{a s}$, A. Mulliria ${ }^{a g}$, R. Nania ${ }^{a i}$, F. Noferini ${ }^{a i}$, F. Nozzoli ${ }^{a t}{ }$, F. Palmonari ${ }^{a i}$, M. Panareo ${ }^{a k}$, M. P. Panetta ${ }^{a k}$, R. Paoletti ${ }^{a h}$, C. Pellegrino ${ }^{a i}$, L. Perasso ${ }^{a f}$, C. Pinto ${ }^{a b}$, G. Piragino $^{a m}$, S. Pisano ${ }^{a e}$, F. Riggi ${ }^{a b}$, G. Righini ${ }^{a}$, C. Ripoli ${ }^{a l}$, M. Rizzi $^{a c}$, G. Sartorelli ${ }^{a i}$, E. Scapparone ${ }^{a i}$, M. Schioppa ${ }^{a u}$, A. Scribano ${ }^{a d}$, M. Selvi ${ }^{a i}$, G. Serri $^{a g}$, S Squarcia ${ }^{a f}$, M. Taiuti ${ }^{a f}$, G. Terreni ${ }^{a d}$, A. Trifirò ${ }^{a q}$, M. Trimarchi ${ }^{a q}$, C. Vistoli ${ }^{r}$, L. Votano ${ }^{a v}$, M. C. S. Williams ${ }^{a i o}$, A. Zichichi ${ }^{a i o}$, R. Zuyeuski ${ }^{a o}$

\footnotetext{
${ }^{a}$ Museo Storico della Fisica e Centro Studi e Ricerche Enrico Fermi, Roma, Italy

${ }^{b}$ INFN and Dipartimento di Fisica e Astronomia "E.Majorana", Università di Catania, Catania, Italy

${ }^{c}$ INFN and Dipartimento Interateneo di Fisica, Università di Bari, Bari, Italy

${ }^{d}$ INFN and Dipartimento di Fisica, Università di Pisa, Pisa, Italy

${ }^{e}$ INFN, Laboratori Nazionali di Frascati, Frascati (RM), Italy

${ }^{f}$ INFN and Dipartimento di Fisica, Università di Genova, Genova, Italy

${ }^{g}$ INFN and Dipartimento di Fisica, Università di Cagliari, Cagliari, Italy

${ }^{h}$ INFN and Dipartimento di Fisica, Università di Siena, Siena, Italy

${ }^{i}$ INFN and Dipartimento di Fisica, Università di Bologna, Bologna, Italy

${ }^{j}$ INFN and Dipartimento di Fisica, Università di Roma Tor Vergata, Roma, Italy

${ }^{k}$ INFN and Dipartimento di Matematica e Fisica, Università del Salento, Lecce, Italy

${ }^{l}$ INFN and Dipartimento di Fisica, Università di Salerno, Salerno, Italy

${ }^{m}$ INFN and Dipartimento di Fisica, Università di Torino, Torino, Italy

${ }^{o}$ CERN, Geneva, Switzerland

PICSC World laboratory, Geneva, Switzerland

${ }^{q}$ INFN Sezione di Catania and Dipartimento di Scienze Matematiche e Informatiche, Scienze

Fisiche e Scienze della Terra, Università di Messina, Messina, Italy

${ }^{r}$ INFN-CNAF, Bologna, Italy

${ }^{s}$ INFN Sezione di Bari, Bari, Italy

${ }^{t}$ Trento Institute for Fundamental Physics and Applications, Trento, Italy

"INFN and Dipartimento di Fisica, Università della Calabria, Cosenza, Italy

${ }^{v}$ INFN, Laboratori Nazionali del Gran Sasso, Assergi (AQ), Italy

E-mail: paola.laroccalct.infn.it
} 
The possibility to observe time correlations between cosmic ray detectors separated by distances much larger than the extent of the highest energy Extensive Air Showers (EAS), i.e. a few km, has been discussed for many years. Although a physical mechanism, based on the photodisintegration of a heavy primary nucleus in the solar field was originally proposed by Gerasimova and Zatsepin in 1960 and later discussed by many authors to justify the existence of such events, the mere observation of long distance time correlations could require by itself novel physics interpretations. A few experimental searches for such events have been reported over the past years, without any definite conclusion reached so far. Although largely depending on many factors, current rate expectations for such events are very low and could stay well below 1 event per year, even with a large detector coverage.

The Extreme Energy Events (EEE) network, based on a relatively large number ( $>50)$ of telescopes with Multigap Resistive Plate Chambers (MRPC), distributed over all the Italian territory, reconstructs cosmic muons with high efficiency and good angular resolution. The wide coverage, number of sites and large time exposure, due to continuous data taking, are unique characteristics of the EEE network, which in principle allow for an investigation of such events. Several strategies are currently employed by the EEE Collaboration to search for long distance time correlations between far detectors, making use of the single-track and multi-track information associated to the various telescopes. A few candidate events with unusually small time differences have been observed, especially from an analysis of multi-track events.

This paper will describe and compare the different strategies adopted so far and will focus on the results of the analysis carried out on a large sample (several years) of data reconstructed by the EEE telescopes.

European Physical Society Conference on High Energy Physics - EPS-HEP2019 -

10-17 July, 2019

Ghent, Belgium

\footnotetext{
* Speaker.
} 


\section{Introduction}

The most recent discoveries in the field of ultra-high energy cosmic rays have been made thanks to the use of extensive surface detector arrays and high resolution air fluorescence detectors. The current generation of detectors has brought definitive improvements in the determination of extensive air shower profiles and the depth of shower maximum, culminating in reliable results on the measurements of cosmic ray energy, composition and arrival directions. In order to achieve a reasonable level of accuracy, the existing ground detector arrays usually cover huge areas (even several thousand square kilometers) with a relatively high granularity, thus being able to sample a single EAS with more detector stations simultaneously involved. A different approach for the study of the cosmic ray radiation has recently emerged. It is based on the use of large sparse arrays of detectors, covering areas of unprecedented size but with a significantly increased detector spacing. The main goal is no longer the accurate study of single EAS, but the search for simultaneous and parallel EAS events, whose existence is still under investigation. The last decades have seen the advent of several experimental projects aimed at the development of sparse networks of cosmic ray detectors that are separated by distances much larger than the extent of the highest energy extensive air showers, typically covering an area equal to that of a nation. One of the most critical aspects of these projects concerns the difficulty of identifying, on such a large spatial scale, a certain number of suitable places where detectors can be installed. In most cases such difficulty has been overcome by placing the detectors in high schools, these buildings being present in large numbers and well distributed on the territory of a nation. This solution has the unique advantage of combining educational and scientific aspects in a single experiment.

In this scenario the Extreme Energy Events Project [1] has built a large network of detectors for cosmic rays installed in Italian high schools. The most challenging goal of this experiment is the search for time correlations between extensive air showers.

A brief description of the EEE Project and its detectors network will be given in the next Section

2. The physics goals of the experiment, with a focus on the search for EAS correlations, are the subject of Section 3. Finally some preliminary results will be described in Section 4.

\section{The detector network of the EEE Project}

The EEE Project, conceived in 2004 by Prof. A. Zichichi, is the flagship project of Museo Storico della Fisica e Centro Studi e Ricerche E. Fermi, with the collaboration of INFN and CERN. It aims at the detection of the secondary cosmic radiation by means of an array of detectors able to reconstruct the direction of the incident charged particles. Currently the network consists of 59 detectors, that were built at CERN by groups of students and teachers and then installed in Italian high schools, with the exception of 6 detectors located in research centers (CERN and INFN Sections). The overall array covers a range from $37.5^{\circ}$ to $46.2^{\circ}$ in latitude, and from $6.1^{\circ}$ to $18.3^{\circ}$ in longitude.

The detectors of the network are telescopes made of three MRPC that were inspired by the Time Of Flight (TOF) detector developed for the ALICE experiment at CERN. The area of the chambers is approximately $90 \times 180 \mathrm{~cm}^{2}$ and they are placed at a relative distance of $0.5 \mathrm{~m}$, as shown in Figure 1. 


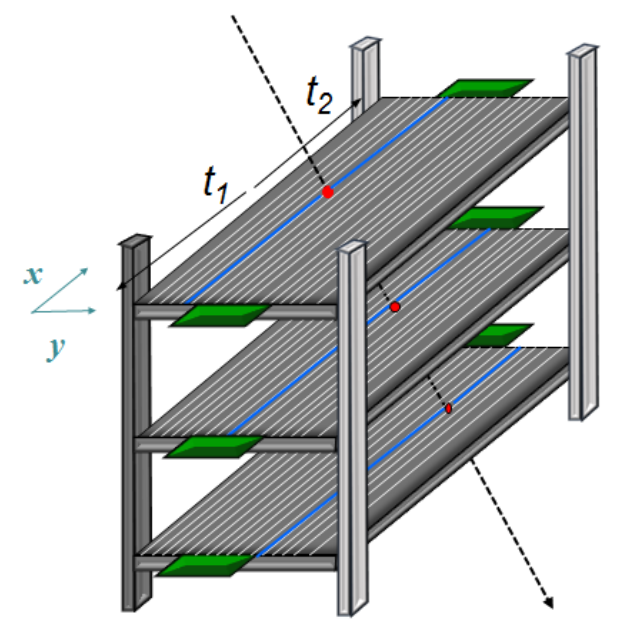

Figure 1: Sketch of a typical EEE telescope.

The performance of the telescopes, in terms of detection efficiency, precision in the measurement of the particles direction and of the TOF between the top and bottom chambers, depends on the MRPC characteristics [2, 3]. Each MRPC consists of a stack of 7 resistive (glass) plates, with high voltage applied only to the external surfaces $( \pm 10 \mathrm{kV})$, that are coated with a special resistive paint. The inner glasses are spaced through a weave made with a $300 \mu \mathrm{m}$ diameter fishing line. The resulting 6 gaps are continuously fluxed with a gas mixture of freon $\mathrm{C}_{2} \mathrm{~F}_{4} \mathrm{H}_{2}(98 \%)$ and $\mathrm{SF}_{6}$ $(2 \%)$, with a rate of $2 \mathrm{l} / \mathrm{h}$. The signal produced by the gas ionization due to the passage of a charged particle through the chamber is induced on the strips electrodes $(2.5 \mathrm{~cm}$ wide with $0.7 \mathrm{~cm}$ pitch), that are laid out on both sides of the stack. The signals propagate to both ends of the MRPC where they are readout by front-end cards: the time difference of the signals arriving at the two strip ends, measured by commercial multi-hit TDCs, provides the particle impact position along the longitudinal direction, while the transverse coordinate is provided by the position of the fired strip. The position resolution, measured on a subset of chambers during the standard data acquisition with cosmics, is of the order of $1 \mathrm{~cm}$ on the transverse coordinate (as expected due to the strip pitch) and $1.5 \mathrm{~cm}$ on the longitudinal coordinate [4]. The plateau efficiency is reached around $18 \mathrm{kV}$ and is better than $90 \%$ for $77 \%$ of the chambers.

Thanks to such performance, each telescope is able to reconstruct the single particle direction with an accuracy of about few degrees. Moreover, the large area of the chambers allows for the simultaneous detection of several particles belonging to the same shower (multi-track events), thus enabling a rough estimate of the shower axis.

All these aspects make the EEE network a unique observatory for the secondary cosmic radiation, not only aimed at the investigation of the phenomena that affect the local cosmic particle flux, but also at the study of the time correlations between EAS.

\section{Scientific goals of the EEE Project}

Since its first beginning in 2004, with a set of 7 pilot sites, the EEE network has grown to become the largest and longest-living MRPC-based telescope array. The whole data set collected 
since fall 2014 reached $10^{11}$ cosmic particle tracks and the network is still expanding, thanks to a recent upgrade activity.

Such a durable and stable data acquisition has been made possible thanks to the contribution of the students and teachers who take care of the operation and maintenance of the telescopes installed at schools. The consequent outreach and educational impact is remarkable since thousands of students are introduced through seminars, lectures and master-classes to the scientific research community, with the opportunity of understanding how a real experiment works, from the infrastructure development to the data acquisition, analysis and publication of scientific results [5].

In addition to its educational feature, the EEE Project was born with ambitious scientific aims and the collaboration has published a large variety of results about the study of the local muon flux and the detection of EAS. Some examples can be found in $[6,7,8,9]$.

The statistics collected so far by the EEE telescopes have recently provided the opportunity to set up the analysis procedures to search for time correlations between cosmic air showers. Indeed, from numerical estimations, the probability to observe these events is extremely low and, as a consequence, a long term data taking is mandatory. The possible physical mechanisms that could justify the existence of long distance correlations suggest a common history of the EAS and lead to rate expectations ranging from $10^{-3}$ to 1 event per $\mathrm{km}^{2}$ per year. More details about the physical mechanisms and numerical estimations can be found in [10] and in the references therein.

\section{Preliminary results on EAS correlations}

To search for possible long distance correlations between cosmic rays, different strategies may be adopted, in order to handle the huge amount of spurious coincidence events between detectors and enhance the probability of observation of rare events. The basic condition is to rely on a pure selection of EAS events. Therefore a first attempt was done limiting the data analysis only to those sites of the network that host at least two close telescopes (i.e. at relative distances within a few $\mathrm{km}$ ) and identifying the EAS from the time and orientation correlations between the telescopes of the same detection site. This approach led to the selection of 5 candidate events of EAS correlations, as reported in [10]. However the EEE network has only 10 sites hosting at least two close telescopes, thus the analyzed data correspond to a small fraction of the whole statistics available.

An alternative strategy exploited was based on the identification of the EAS by selecting multitrack events (i.e. number of tracks per event $>=3$ ) in each telescope of the network. The whole statistics from 2013 to 2018 was analyzed looking at all the possible pair combinations between the 42 telescopes in data acquisition. Basic cuts were applied on the quality of multi-track events (more precisely on the $\chi^{2}$ and parallelism of the tracks) and on the number of the tracks per event $\left(n_{\text {tracks }}>3\right)$. The number of correlated events observed as a function of the coincidence time window is shown in 2 (left) and compared to the expected spurious background (red line). With this method, for coincidence time windows of the order of $10^{-5}-10^{-4} \mathrm{~s}$, we obtained 40 events arising from all telescope combinations, with an expected number of spurious events equal to 23.4, roughly corresponding to a $3 \sigma$ effect.

This study takes into consideration only the possibility to observe coincidences between at most two distant detection sites. Thus we extended our analysis considering the possibility to observe time correlation events that simultaneously involve a large number of telescopes. An au- 

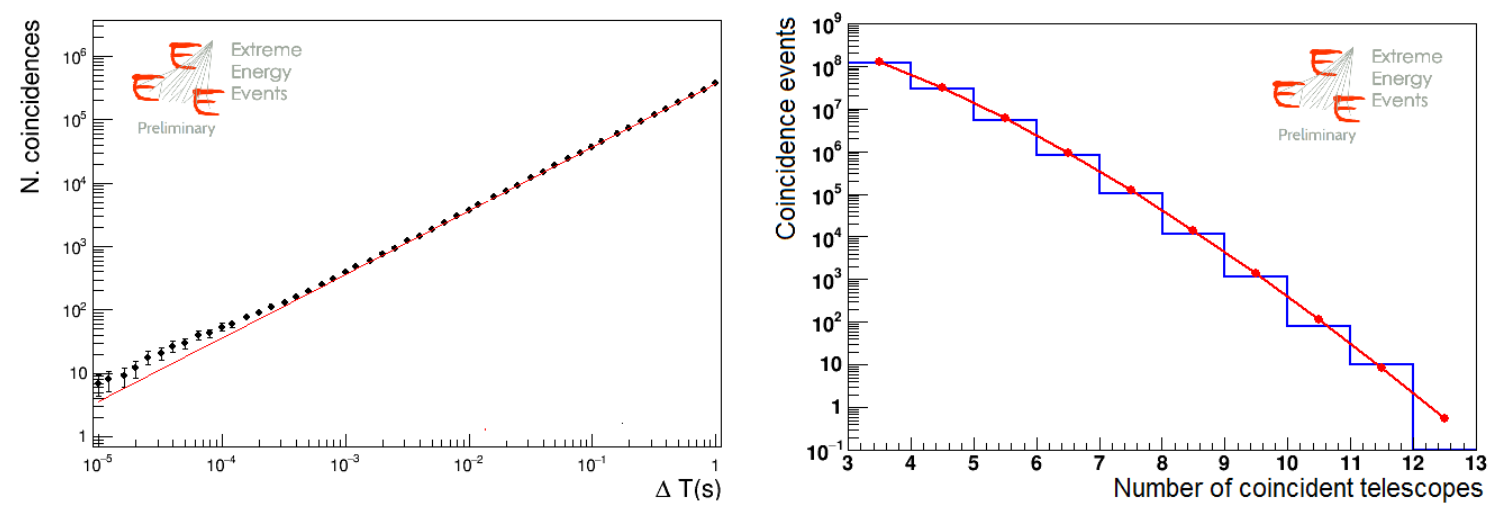

Figure 2: Left: number of coincident events (in black) as a function of the time window, compared with the expected number of accidental coincidences in red. Right: number of telescopes simultaneously fired in a $\pm 1 \mathrm{~ms}$ time window (blue line), compared with the expected number of accidental coincidences (red points).

tomatic procedure was setup in order to evaluate, in each time window of $\pm 1 \mathrm{~ms}$, how many telescopes are simultaneously detecting a particle. Figure 2 (right) shows the distribution of the number of telescopes simultaneously fired in $\pm 1 \mathrm{~ms}$ time window. Even if high multiplicity events (up to 12 telescopes) were observed, the number of experimental coincidence events are compatible with the expected level of spurious coincidences (in red), that was estimated knowing the number of telescopes taking data, their individual rates and their efficiency. It is worth to mention that the plot in Figure 2 (right) refers to a small data set (only 1 month) and the analysis of the whole statistics is still ongoing.

In conclusion, first analyses of the dataset of cosmic ray events detected by EEE telescopes have shown the capability of this network to search for rare events such as time correlations between cosmic air showers. Even if few candidate events were observed, a clear evidence for long distance correlations needs higher statistics. Further work on this subject will proceed, deeply investigating and debugging the results already obtained and taking into account the additional statistics which will come from the upcoming data taking runs.

\section{References}

[1] EEE Project web site: https://eee.centrofermi.it

[2] M. Abbrescia et al. (EEE Collaboration), Nucl. Inst. and Meth. A845(2017)383

[3] F. Carnesecchi et al. (EEE Collaboration), PoS(EPS-HEP2017)820

[4] M. Abbrescia et al. (EEE Collaboration), JINST 13(2018)P08026

[5] S. Grazzi et al. (EEE Collaboration), PoS(EPS-HEP2017)823

[6] M. Abbrescia et al. (EEE Collaboration), Nucl. Inst. and Meth. A816 (2016) 142

[7] M. Abbrescia et al. (EEE Collaboration), PoS(ICRC 2015)097

[8] M. Abbrescia et al. (EEE Collaboration), Eur. Phys. J. Plus 130(2015)187

[9] M. Abbrescia et al. (EEE Collaboration), Eur. Phys. J. Plus 128(2013)148

[10] M. Abbrescia et al. (EEE Collaboration), Eur. Phys. J. Plus 133(2018)34 